13

\title{
Влияние толщины пленки Pt на процессы роста зерен при ее отжиге
}

\author{
(c) Р.В. Селюков, В.В. Наумов, С.В. Васильев \\ Ярославский фрилиал Физико-технологического института РАН, \\ 150007 Ярославль, Россия \\ e-mail: rvselyukov@mail.ru
}

(Поступило в Редакцию 23 октября 2017 г.)

Пленки Pt с толщиной $h=20-100 \mathrm{~nm}$, нанесенные на окисленную пластину $c$-Si $(100)$, подвергались отжигу в вакууме в режиме $500^{\circ} \mathrm{C} / 1 \mathrm{~h}$, в результате которого произошли рекристаллизация и рост зерен. Одновременно с нормальным наблюдался и аномальный рост, что привело к разделению зерен на фракции, соответственно, обычных и вторичных зерен. Для $h=20-40 \mathrm{~nm}$ вторичные зерна становятся заметно крупнее обычных, поэтому распределение латеральных размеров зерен становится бимодальным. Найдено, что скорость аномального роста латеральных размеров зерен увеличивается с уменьшением $h$, тогда как скорость нормального роста не зависит от $h$. С помощью анализа профилей рентгенодифракционных максимумов $\operatorname{Pt}(111)$ и $\operatorname{Pt}(222)$ найдено, что в результате отжига средний размер областей когерентного рассеяния $D$ увеличивается. Для пленок, подвергнутых отжигу, $D$ сублинейно увеличивается с ростом $h$, тогда как для исходных пленок наблюдается линейный рост $D$.

DOI: 10.21883/JTF.2018.06.46027.2526

\section{Введение}

Тонкие поликристаллические пленки Pt применяются в качестве тыльного электрода в приборах с перовскитоподобными сегнетоэлектриками [1-3], в качестве катализатора в сенсорах различных газов [4,5], как покрытия с низкой поглощательной способностью в ИК диапазоне [6], а также в качестве резистивных нагревателей и сенсоров температуры в МЭМС [7]. Во всех этих случаях микроструктура Pt оказывает влияние на характеристики приборов и покрытий. Для перехода материала в сегнетоэлектрическую фазу его после нанесения на Pt электрод отжигают при $500-700^{\circ} \mathrm{C}$, а сенсоры, защитные покрытия и нагреватели во время работы длительное время находятся при высоких температурах. Таким образом, во всех вышеперечисленных областях применения пленки Pt подвергаются нагреву, который меняет их микроструктуру. Общими для объемных материалов и пленок являются процессы рекристаллизации и последующего роста зерен во время отжига, если его температура превышает определенное значение. При рекристаллизации происходит перестройка зерен, приводящая к релаксации напряжений и уменьшению микродеформаций. В процессе роста зерен наряду с так называемым нормальным ростом может наблюдаться аномальный рост, характеризующийся быстрым увеличением размеров некоторых зерен, которые принято называть вторичными [8]. Более медленный нормальный рост характерен для основной фракции зерен, которые принято называть обычными [8]. Таким образом, если в системе с исходно одномодальным распределением размеров зерен в процессе отжига имеет место аномальный их рост, то распределение становится бимодальным, в случае только нормального роста оно остается одномодальным. В общем случае аномальный рост зерен обусловлен уменьшением суммы поверхностной энергии (которая складывается из энергии поверхности пленки и энергии границы раздела пленки и подложки) и энергии межзеренных границ [8]. Для тонких пленок условие минимизации поверхностной энергии является более значимым, чем для объемных материалов, из-за большего отношения площади поверхности к объему, что имеет два важных следствия. Для вторичных зерен в пленках, как правило, характерна ориентация параллельно подложке тех атомных плоскостей, которые обеспечивают минимальную поверхностную энергию $[9,10]$, например, для ГЦК металлов это плоскости (111). Кроме того, теория аномального роста, учитывающая влияние поверхностной энергии, предсказывает увеличение скорости роста латеральных размеров вторичных зерен с уменьшением толщины пленки [8], что было подтверждено экспериментально для пленки $\mathrm{Au}$ [11]. Также необходимо отметить, что рекристаллизация и рост зерен в пленках наблюдаются при температурах, заметно меньших, чем в соответствующих объемных материалах [9,11-14]. Аномальный рост наблюдался в пленках, в том числе, ГЦК металлов: $\mathrm{Au}[9,11]$, $\mathrm{Cu}[12,13], \mathrm{Ag}[15], \mathrm{Pt}[16,17]$. В частности, для $\mathrm{Au}$ исследовалась зависимость скорости роста вторичных зерен от толщины пленки, аналогичных исследований для пленок Pt авторами найдено не было.

Таким образом, одинаковая термообработка пленок разной толщины приводит к существенно разным изменениям их микроструктуры. Это необходимо учитывать при изготовлении и эксплуатации упомянутых выше приборов и покрытий с пленками Pt. Целью данной работы было установить, как в зависимости от толщины пленки Pt меняется воздействие отжига в вакууме в режиме $500^{\circ} \mathrm{C} / 1 \mathrm{~h}$ на микроструктуру Рt. Для этого до и после отжига проводились измерения средних латеральных размеров зерен, размеров областей когерентного рассеяния (ОКР) и микродеформаций. 

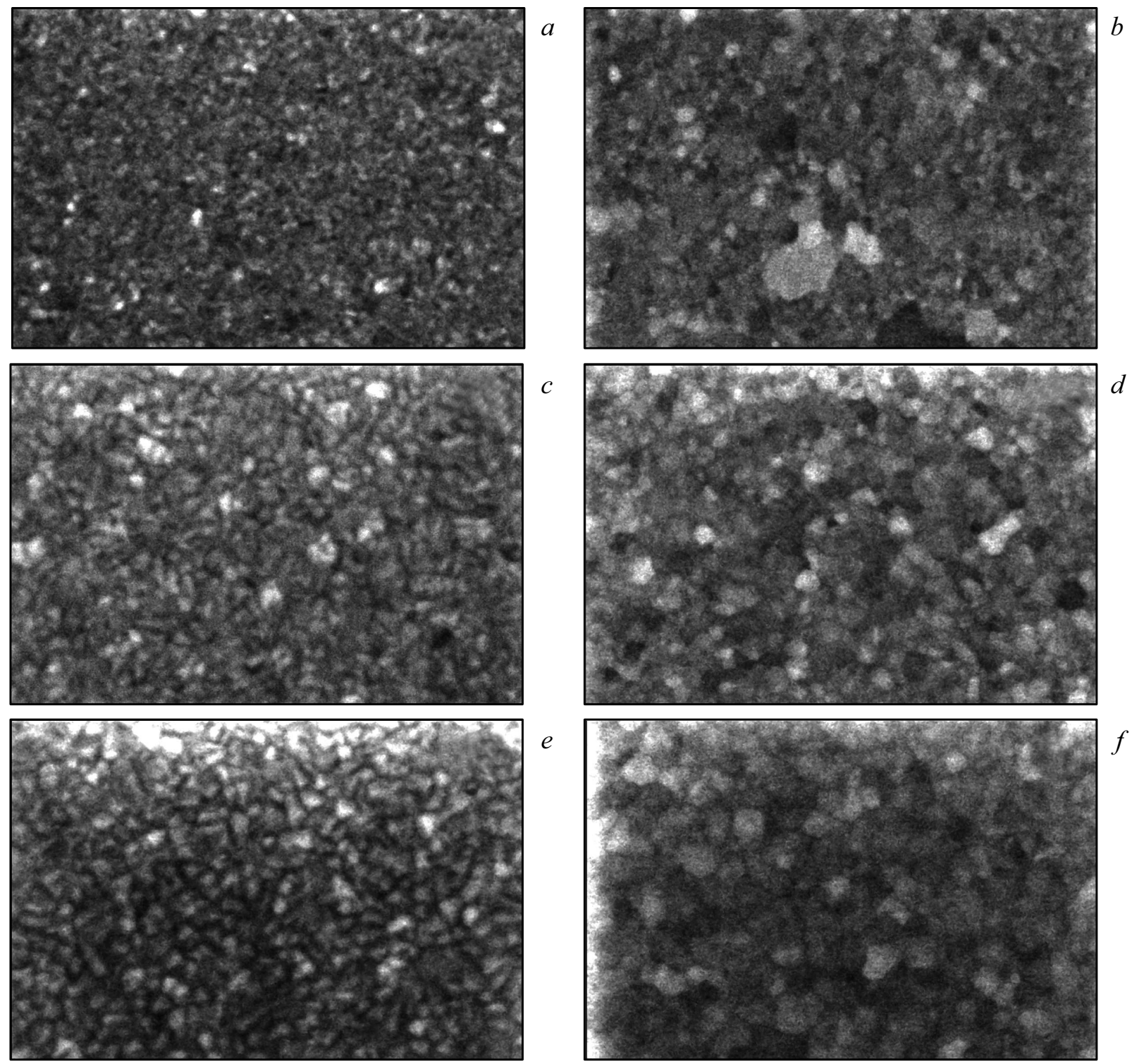

Рис. 1. СЭМ изображения пленок Рt исходных $(a, c, e)$ и после отжига $500^{\circ} \mathrm{C} / 1 \mathrm{~h}(b, d, f)$. Толщины пленок: $20(a, b), 60(c, d)$, $100 \mathrm{~nm}(e, f)$. Размер изображения по горизонтали $-580 \mathrm{~nm}$.

\section{1. Эксперимент}

Пленки Pt толщинами 20-100 nm наносились методом магнетронного распыления на окисленную пластину $\mathrm{Si}(100)$. Нанесение пленки проводилось в установке SCR 651 „Tetra“ (Alcatel) при комнатной температуре, остаточном давлении $5 \cdot 10^{-5} \mathrm{~Pa}$ и давлении аргона 0.2 Ра. Скорость нанесения пленки Pt составляла $0.5 \mathrm{~nm} / \mathrm{s}$. После нанесения пленки отжигались в вакууме при $500^{\circ} \mathrm{C}$ в течение $1 \mathrm{~h}$. Данная температура примерно соответствует порогу рекристаллизации в объемной Pt [14].

Микроструктура пленок до и после отжига исследовалась методами рентгеноструктурного анализа и сканирующей электронной микроскопии (СЭМ). На дифракто- метре ARL X'tra (Thermo Fisher Scientific) проводились съемка $\theta-2 \theta$-дифрактограмм. При съемке шаг сканирования был равен $0.02^{\circ}$, скорость сканирования составляла $0.15^{\circ} / \mathrm{min}$. Использовалась рентгеновская трубка с медным анодом. Линия $\mathrm{Cu} K_{\beta}$ устранялась выбором порогов дискриминатора амплитуды импульсов, поступающих с детектора. Профили дифракционных максимумов исходно представляли собой сумму $\mathrm{Cu} K_{\alpha 1^{-}}$и $\mathrm{Cu} K_{\alpha 2}$-компонент, $\mathrm{Cu} K_{\alpha 2}$-компонента удалялась методом Речингера. Размеры ОКР и величины микродеформаций определялись с помощью анализа профилей дифракционных максимумов $\mathrm{Pt}(111)$ и (222), проведенного по методу аппроксимаций. Аппаратная функция определялась методом аппроксимации профилей дифракционных максимумов эталона NIST SRM 1976а. СЭМ изображе- 

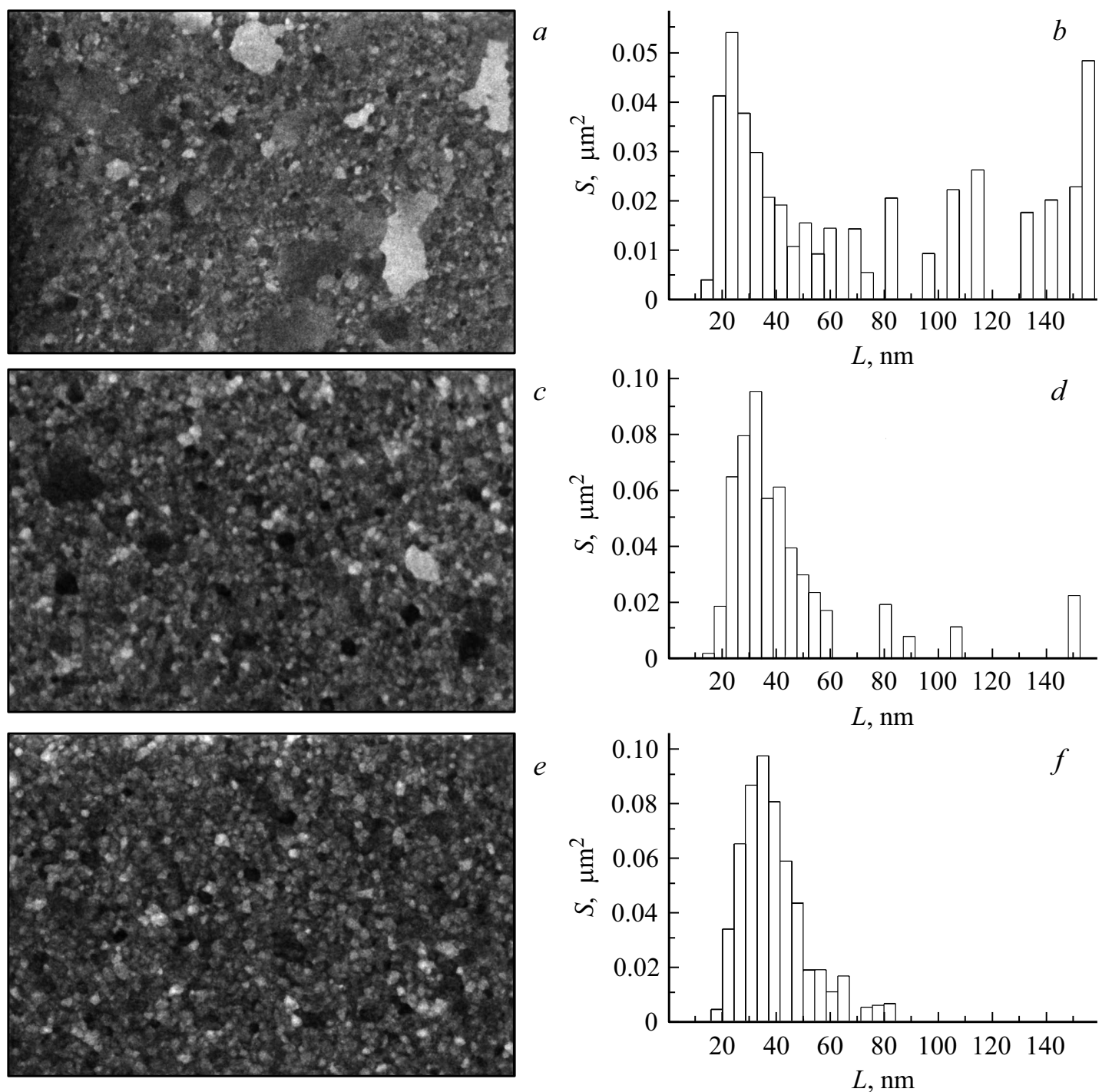

Рис. 2. СЭМ изображения пленок $\mathrm{Pt}$ после отжига $(a, c, e)$ и соответствующие гистограммы распределения площадей $S$, которые занимают фракции зерен с латеральным размером $L(b, d, f)$. Толщины пленок: $20(a, b), 40(c, d), 60 \mathrm{~nm}(e, f)$. Размер изображения по горизонтали $-1.17 \mu \mathrm{m}$.

ния были получены с помощью микроскопа Supra-40 (Carl Zeiss). Распределения латеральных размеров зерен были получены путем обработки СЭМ изображений с помощью программы JMicroVision. Толщина пленок измерялась по сколу с помощью СЭМ. Химический состав пленок исследовался методом энергодисперсионной рентгеновской спектроскопии (EDX) с помощью приставки INCAx-act (Oxford Instruments).

\section{2. Результаты и обсуждение}

EDX-измерения не показали наличия загрязнений в пленках Pt, кроме того, энергодисперсионные спектры образцов до и после отжига были одинаковыми. Это дает основание утверждать, что вторичные зерна, наблюдаемые в пленках после отжига, не являются загрязнениями.

\section{1. Анализ распределений латеральных размеров зерен пленок Pt}

На рис. 1 и 2 приведены СЭМ изображения поверхности пленок $\mathrm{Pt}$, исходных и после отжига. Несколько выводов можно сделать уже на основании визуального анализа. Во всех исходных пленках аномально крупных зерен не наблюдается, и распределение латеральных размеров зерен $L-$ одномодальное. В пленках, подвергнутых отжигу, толщиной $h=60-100 \mathrm{~nm}$ отсутствуют вторичные зерна, и распределение $L-$ также одномодальное. На рис. 2 видно, что при уменьшении $h$ до $20-40 \mathrm{~nm}$ в пленках после отжига появляются вторичные зерна, заметно более крупные, чем зерна основной фракции, таким образом, в этом случае распределение $L$ является бимодальным. Эти результаты согласуются с данными работы [16], где наблюдался аномальный рост зерен в пленке $\mathrm{Pt}$, осажденной на $\mathrm{Si}_{3} \mathrm{~N}_{4}$, после ее 

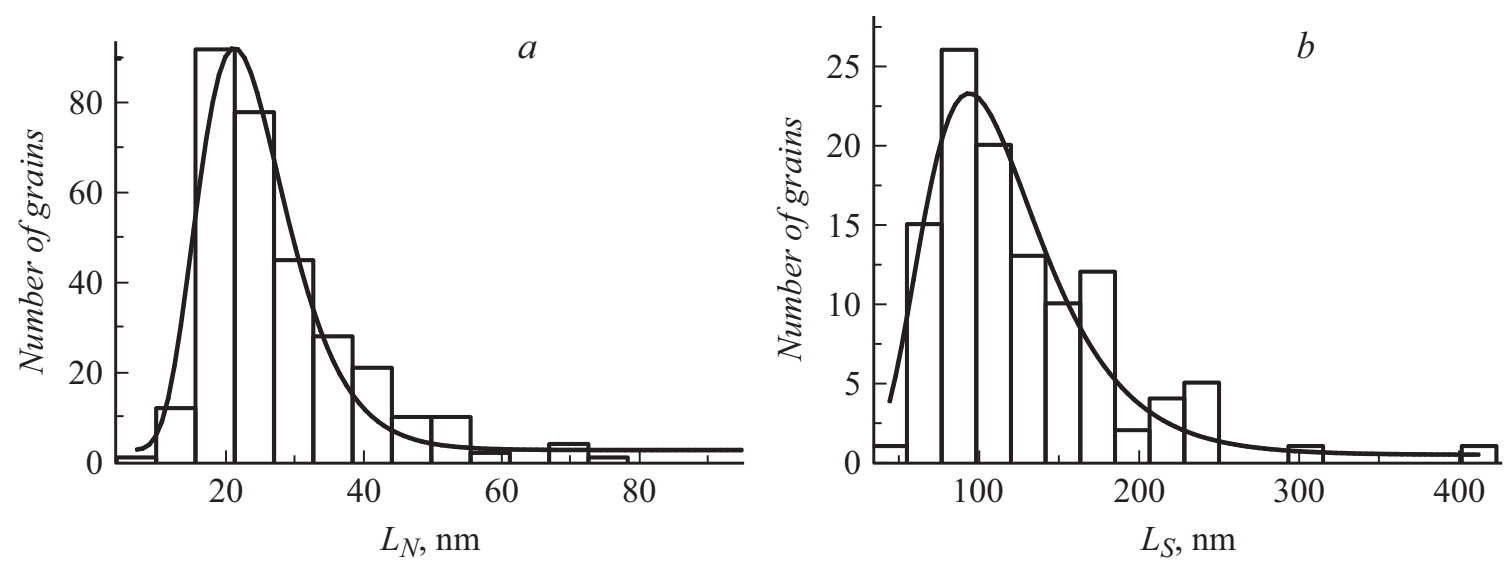

Рис. 3. Распределения латеральных размеров обычных зерен $L_{N}(a)$ и вторичных зерен $L_{S}(b)$ для пленки $20 \mathrm{~nm}$ после отжига (гистограммы) и их аппроксимации логнормальной зависимостью.

отжига при температурах $500-830^{\circ} \mathrm{C}$. Таким образом, для пленок, подвергнутых отжигу, необходимо ввести разделение зерен на фракции обычных зерен с латеральными размерами $L_{N}$ и вторичных зерен с латеральными размерами $L_{S}$. Так как в пленках $60-100 \mathrm{~nm}$ после отжига крупных зерен не наблюдается, все зерна этих пленок будут рассматриваться как обычные. Зерна исходной пленки будут именоваться исходными с латеральными размерами $L_{I}$. На рис. 2 показано, что с уменьшением $h$ происходит увеличение среднего латерального размера вторичных зерен $\left\langle L_{S}\right\rangle$, а доля поверхности, занимаемая вторичными зернами, для пленки $20 \mathrm{~nm}$ больше, чем для пленки $40 \mathrm{~nm}$. Также из рис. 1 видно, что для пленок до и после отжига $\left\langle L_{I}\right\rangle$ и $\left\langle L_{N}\right\rangle$ увеличиваются с ростом $h$. Наконец, для каждой $h\left\langle L_{N}\right\rangle$ для пленки после отжига больше, чем $\left\langle L_{I}\right\rangle$ для исходной пленки.

С помощью СЭМ изображений для каждой пленки путем измерения $L$ всех четко наблюдаемых зерен были рассчитаны распределения $L_{I}, L_{N}$ и $L_{S}$, а также вычислены средние значения $\left\langle L_{I}\right\rangle,\left\langle L_{N}\right\rangle$ и $\left\langle L_{S}\right\rangle$. Для каждого образца проводилась обработка суммарно $\sim 10^{3}$ зерен на 2-4 изображениях. Концентрация обычных зерен много больше, чем вторичных, поэтому распределение $L_{S}$ трудно выделить из общего бимодального распределения $L$. Поэтому для нахождения распределений $L_{S}$ и $L_{N}$ использовались СЭМ изображения с меньшим и большим увеличением соответственно. В первом случае можно было четко наблюдать границы только вторичных зерен, поэтому обычные зерна не измерялись. Во втором случае в кадр, как правило, попадало несколько вторичных зерен (рис. 1), которые в расчетах не учитывались. Было найдено, что во всех случаях распределения $L_{I}$, $L_{N}$ и $L_{S}$ удовлетворительно описываются логнормальной зависимостью, имеющей следующий вид [11]:

$$
N \propto \frac{1}{w L} \exp \left[-\frac{\ln ^{2}\left(L / L_{0}\right)}{2 w^{2}}\right],
$$

где $w$ - дисперсия величины $\ln (L)$. На рис. 3 представлены распределения $L_{N}$ и $L_{S}$ для пленки $20 \mathrm{~nm}$ после

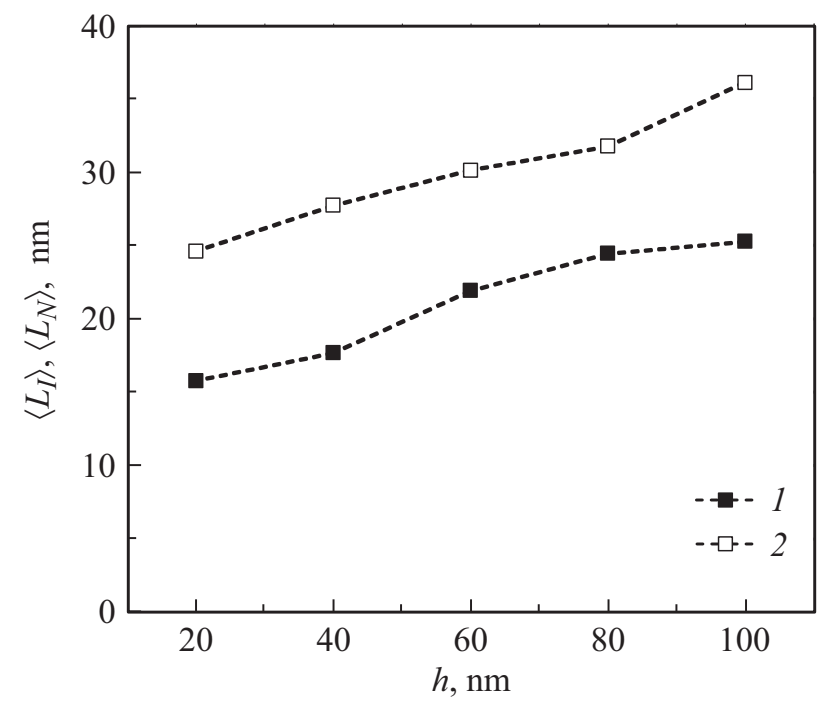

Рис. 4. Зависимости среднего латерального размера исходных зерен $\left\langle L_{I}\right\rangle(1)$ и обычных зерен $\left\langle L_{N}\right\rangle(2)$ от толщины пленки $h$.

отжига. На рис. 2 для пленок, подвергнутых отжигу, представлены гистограммы распределения площадей $S$, которые занимают фракции зерен с латеральным размером $L$. Видно, что для $h=60 \mathrm{~nm}$ вторичные зерна отсутствуют, при $h=40 \mathrm{~nm}$ они занимают незначительную долю площади, наконец, для $h=20 \mathrm{~nm}$ вторичные зерна занимают площадь, сопоставимую с таковой для обычных зерен, что согласуется со сделанными выше качественными выводами. Было найдено, что для $h=20 \mathrm{~nm}$ $\left\langle L_{S}\right\rangle$ равен $116 \mathrm{~nm}$, для $h=40 \mathrm{~nm}\left\langle L_{S}\right\rangle$ равен $87 \mathrm{~nm}$. Визуально представляется, что для пленки $20 \mathrm{~nm}\left\langle L_{S}\right\rangle$ равен $\sim 150-200 \mathrm{~nm}$, но это обусловлено наличием нескольких больших $(200-400 \mathrm{~nm})$ зерен из хвоста распределения, основная же масса вторичных зерен заметно меньше. Рост $\left\langle L_{S}\right\rangle$ с уменьшением $h$ согласуется с теорией [8], а также с результатами работы [17], где пленка Pt $150-250 \mathrm{~nm}$ на подслое Ті подвергалась 
отжигу при $750^{\circ} \mathrm{C}$, и работы $[11]$, где наблюдался аномальный рост зерен в пленке $\mathrm{Au}$ при комнатной температуре. Таким образом, можно утверждать, что для данной микроструктуры исходных пленок $\mathrm{Pt}$ и для данных условий отжига при $h \sim 40 \mathrm{~nm}$ скорость роста вторичных зерен начинает заметно превышать скорость роста обычных зерен.

На рис. 4 представлены зависимости $\left\langle L_{I}\right\rangle$ и $\left\langle L_{N}\right\rangle$ от $h$ для исходных и подвергнутых отжигу пленок соответственно, которые подтверждают сделанные выше качественные выводы относительно исходных и обычных зерен. Рост $\left\langle L_{I}\right\rangle$ и $\left\langle L_{N}\right\rangle$ с увеличением $h$ в обоих случаях можно охарактеризовать как линейный. Линейный рост в случае исходной пленки согласуется с результатами для пленки Ni [18], также рост $\left\langle L_{I}\right\rangle$ с увеличением $h$ для пленки Pt наблюдался в [19-21]. В случае пленки, подвергнутой отжигу, линейное увеличение $\left\langle L_{N}\right\rangle$ согласуется с результатами для пленки $\mathrm{Au}$ [9]. Можно видеть, что разность $\left\langle L_{N}\right\rangle-\left\langle L_{I}\right\rangle$ примерно одинакова для всех $h$, это позволяет утверждать, что скорость нормального роста зерен не зависит от $h$.

\section{2. Рентгеноструктурный анализ пленок Pt}

До и после отжига на $\theta-2 \theta$-дифрактограммах образцов присутствуют пики $\mathrm{Pt}(111)$ и (222), а также слабые пики $\operatorname{Pt}(200)$ и (311), что свидетельствует о наличии (111) текстуры. Для всех исходных образцов межплоскостное расстояние $d_{(111)}$, измеренное вдоль нормали к поверхности пленки, было больше, чем значение $d_{(111)}$, измеренное для фрагмента мишени $\mathrm{Pt}$, в котором с помощью отжига в вакууме были устранены механические напряжения. Таким образом, деформация растяжения пленки вдоль нормали к поверхности свидетельствует о сжимающих напряжениях, действующих в плоскости пленки. После отжига образцов $d_{(111)}$ во всех случаях становится меньше, чем $d_{(111)}$ фрагмента мишени $\mathrm{Pt}$, следовательно, напряжения, действующие в плоскости пленки, становятся растягивающими. Таким образом, в результате отжига напряжения сжатия устраняются, а напряжения растяжения возникают при охлаждении образца из-за того, что температурный коэффициент линейного расширения (ТКЛР) Pt больше, чем ТКЛР $\mathrm{SiO}_{2}$ [10]. Релаксация напряжений является свидетельством прошедшей во всех пленках рекристаллизации.

Расчет средних значений микродеформаций $\varepsilon$ и размеров ОКР $D$ проводился с помощью метода аппроксимаций [22]. Известно, что форма и ширина наблюдаемых на рентгенограммах профилей дифракционных максимумов определяются как инструментальными причинами, так и микроструктурой исследуемых объектов. Для описания физического уширения профилей тонких пленок необходимо учитывать как конечность размеров ОКР (дисперсность), так и микродеформации кристаллической решетки. В рамках метода аппроксимаций каждая инструментальная и физическая причина, влияющая на форму профиля, описывается некоторой известной функцией, а наблюдаемый профиль является сверткой всех этих функций [23]. Из профилей $\mathrm{Pt}(111)$ и (222) по методу Речингера были выделены $K_{\alpha 1}$-компоненты, и было найдено, что они удовлетворительно аппроксимируются функцией Фогта $V(2 \theta)$ (рис. 5), представляющей собой свертку функций Гаусса и Коши:

$$
V\left(2 \theta, \beta_{G}, \beta_{C}\right)=\int_{-\infty}^{+\infty} \frac{\exp \left(-\pi x^{2} / \beta_{G}^{2}\right)}{1+\pi^{2}(2 \theta-x)^{2} / \beta_{C}^{2}} d x
$$

где $\beta_{G}$ и $\beta_{C}-$ интегральная ширина профиля, описываемого соответственно функцией Гаусса и Коши. Исключение составляет пленка $20 \mathrm{~nm}$ после отжига, чей профиль $\mathrm{Pt}(111)$ потребовал отдельного анализа. Также было найдено, что аппаратная функция дифрактометра удовлетворительно аппроксимируется функцией $V(2 \theta)$. Так как свертка двух функций Фогта есть функция Фогта [23], то в качестве аппроксимации наблюдаемого и физического профиля, а также аппаратной функции была использована $V(2 \theta)$, а $\beta_{G}$ и $\beta_{C}$ наблюдаемого профиля определяются выражениями [23]

$$
\begin{aligned}
& \beta_{C}=B_{C}+b_{C}, \\
& \beta_{G}^{2}=B_{G}^{2}+b_{G}^{2},
\end{aligned}
$$

где $B_{G}$ и $B_{C}-$ интегральная ширина соответственно компонент Гаусса и Коши физического профиля, $b_{G}$ и $b_{C}$ - интегральная ширина компонент Гаусса и Коши аппаратной функции. Принято считать, что функция Коши физического профиля описывает вклад дисперсности [24], таким образом, $B_{C}$ и $D$ связаны формулой Шерpepa [22]

$$
B_{C}^{(h k l)}=\frac{\lambda}{D \cos \theta_{(h k l)}},
$$

где $\lambda$ - длина волны рентгеновского излучения (в данном случае, $K_{\alpha 1}$-линии $\left.\mathrm{Cu}\right), \theta_{(h k l)}-$ половинный угол дифракции на семействе плоскостей $(h k l)$.

Также принято считать, что функция Гаусса физического профиля описывает вклад микродеформаций [24], поэтому $B_{G}$ и $\varepsilon$ связаны известным соотношением [22]

$$
B_{G}^{(h k l)}=4 \varepsilon \tan \theta_{(h k l)} .
$$

Интегральная ширина профиля по определению равна

$$
\beta=\frac{\int_{-\infty}^{+\infty} V\left(2 \theta, \beta_{G}, \beta_{C}\right) d 2 \theta}{V\left(0, \beta_{G}, \beta_{C}\right)}=\frac{\beta_{G} \beta_{C}}{V\left(0, \beta_{G}, \beta_{C}\right)} .
$$

Выражение (7) является уравнением, связывающим $D$ и $\varepsilon$. Для нахождения $D$ и $\varepsilon$ необходимо решить систему из двух таких уравнений, взятых для двух порядков отражения от одного и того же семейства атомных 

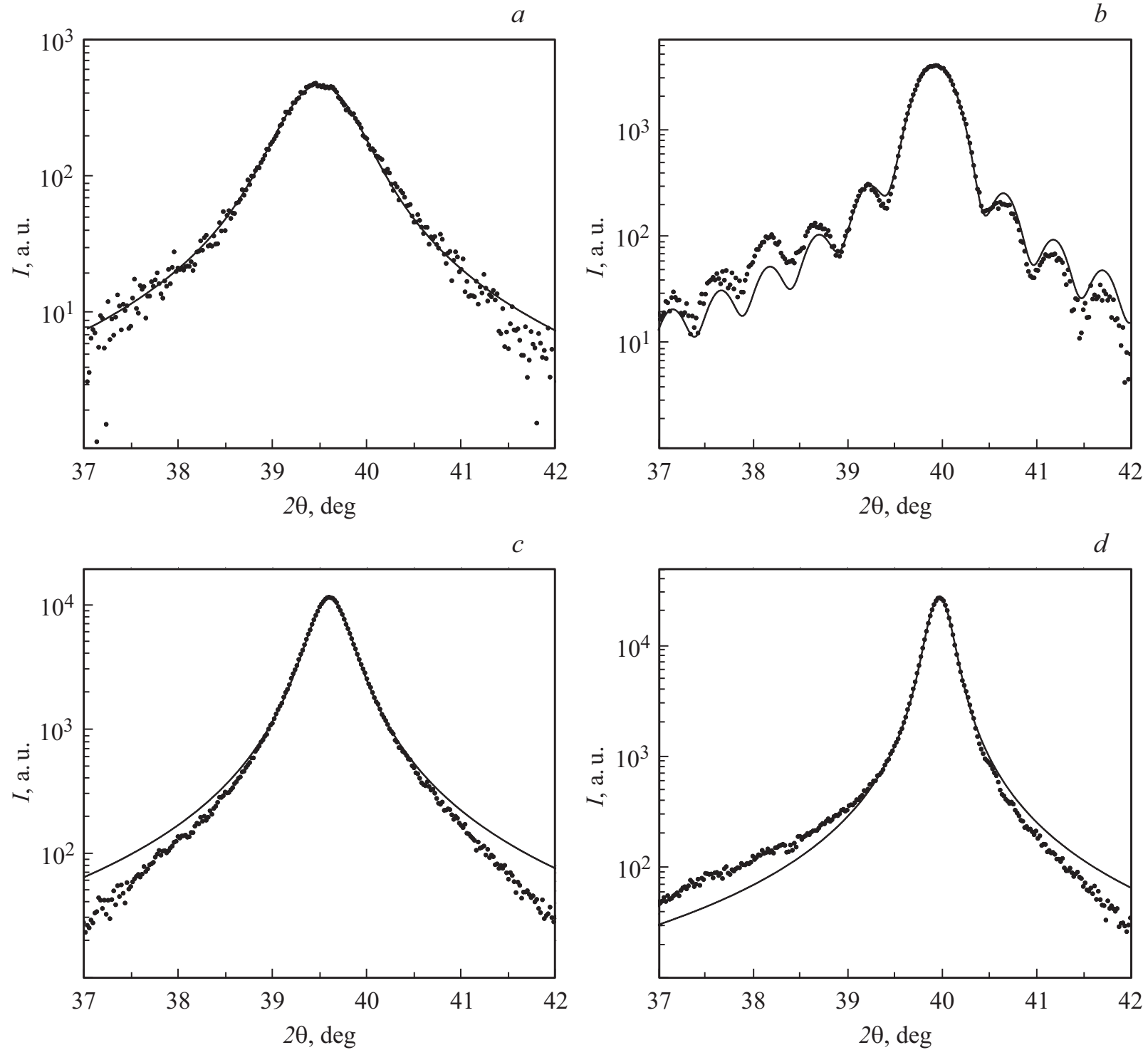

Рис. 5. $K_{\alpha 1}$-компоненты дифракционных профилей $\operatorname{Pt}(111)$ пленок $20(a, b)$ и $100 \mathrm{~nm}(c, d)$, исходных $(a, c)$ и после отжига $(b, d)$. Точки - экспериментальные данные, сплошные линии - их аппроксимация функцией Фогта $(a, c, d)$ или суммой функций Лауэ и Коши $(b)$.

плоскостей, в данном случае для профилей $\operatorname{Pt}(111)$ и (222) $[22,24]$

$$
\left\{\begin{array}{l}
\beta^{(111)}=\beta_{G}^{(111)} \beta_{C}^{(111)} / V\left(0, \beta_{G}^{(111)}, \beta_{C}^{(111)}\right) \\
\beta^{(222)}=\beta_{G}^{(222)} \beta_{C}^{(222)} / V\left(0, \beta_{G}^{(222)}, \beta_{C}^{(222)}\right),
\end{array}\right.
$$

где $\beta^{(111)}$ и $\beta^{(222)}-$ интегральная ширина профиля (111) и (222) соответственно.

На профиле $\operatorname{Pt}(111)$ пленки $20 \mathrm{~nm}$, подвергнутой отжигу, наблюдаются осцилляции. Такие осцилляции наблюдаются обычно, когда распределение ОКР по размерам становится узким [10,23], вклад дисперсности в данном случае принято описывать функцией Лауэ [23]. Аппроксимация $K_{\alpha 1}$-компоненты профиля пленки $20 \mathrm{~nm}$ одной функцией Лауэ неудовлетворительна, лучший результат дает аппроксимация суммой функций Коши и Лауэ (рис. 5, b):

$$
\begin{aligned}
I(2 \theta)= & \frac{A_{C}}{1+\left[\pi\left(2 \theta-2 \theta_{C}\right) / \beta_{C}\right]^{2}} \\
& +A_{L}\left\{\frac{\sin \left[\pi\left(2 \theta-2 \theta_{L}\right) / \beta_{L}\right]}{\pi\left(2 \theta-2 \theta_{L}\right) / \beta_{L}}\right\}^{2}
\end{aligned}
$$

со следующими значениями параметров: $2 \theta_{C}=39.86^{\circ}$, $2 \theta_{L}=39.94^{\circ}, \beta_{C}=0.99^{\circ}, \beta_{L}=0.51^{\circ}, A_{C}=721, A_{L}=$ $=3366$. Из-за пренебрежения вкладами микродеформаций и аппаратной функции данные значения носят оценочный характер. Исключением является $\beta_{L}$, так как этот параметр определяет период осцилляций и поэтому 
$a$

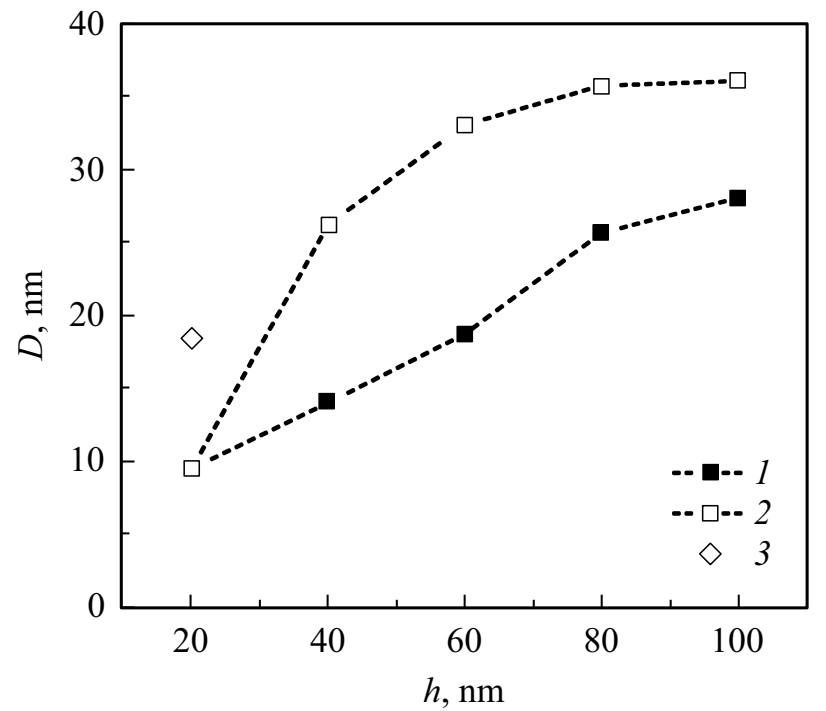

$b$

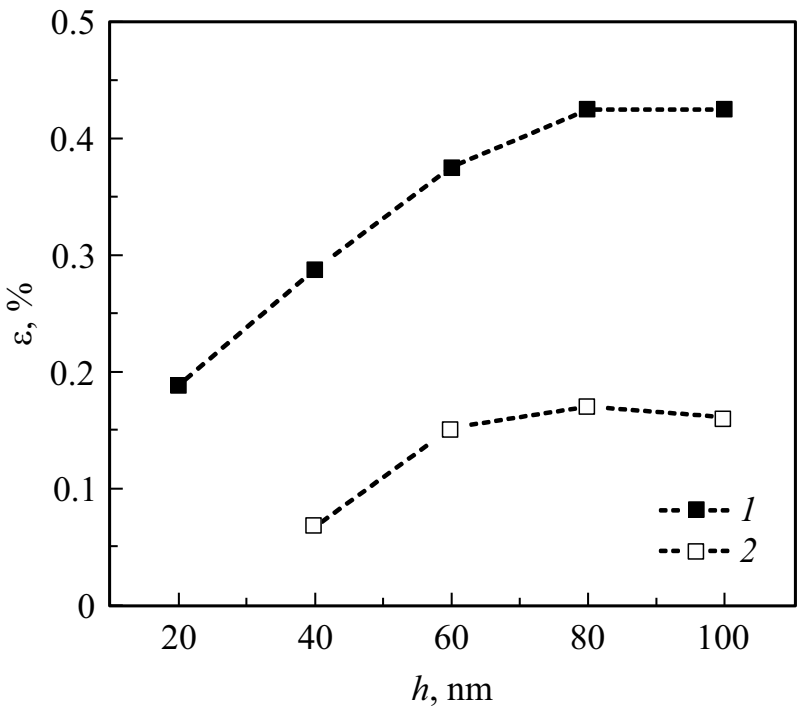

Рис. 6. Зависимости среднего размера ОКР $D(a)$ и среднего значения микродеформаций $\varepsilon(b)$ от толщины пленки $h$. $1-$ исходная пленка, $2-$ пленка после отжига, $3-D$ вторичных зерен для пленки $20 \mathrm{~nm}$.

может быть вычислен с высокой точностью. Описание профиля суммой двух функций можно объяснить присутствием в Pt зерен двух типов. Если, пользуясь формулой Шеррера, вычислить $D$ для обоих профилей, то получим: $D_{L}=19 \mathrm{~nm}, D_{C}=10 \mathrm{~nm}$. Можно утверждать, что вклад в виде функции Лауэ дают вторичные зерна, а вклад в виде функции Коши - обычные зерна. Как указывалось выше, наблюдение осцилляций возможно только в случае малого разброса значений $D_{L}$, который достигается следующим образом. Вторичные зерна в процессе роста поглощают окружающий материал, в результате чего их высоты становятся равными толщине пленки в участке роста, а разброс их высот будет определяться шероховатостью пленки и, очевидно, будет меньше разброса высот обычных зерен. При этом СЭМ измерения показывают, что шероховатость пленки $20 \mathrm{~nm}$ до отжига много меньше ее толщины и не меняется заметно в результате отжига. Таким образом, не наблюдается заметного роста высот вторичных зерен, а среднее значение высоты зерна оказывается равным $D_{L}$, что говорит о высокой степени их структурного совершенства. То, что осцилляции не наблюдаются на более толстых пленках, подвергнутых отжигу, можно объяснить меньшей долей материала во вторичных зернах. Таким образом, согласно данным рентгеноструктурного анализа, долю материала во вторичных зернах пленки $20 \mathrm{~nm}$ можно считать значительной, что соответствует данным CЭМ. $D$ и $\varepsilon$ остальных пленок были вычислены путем решения системы (8), результаты представлены на рис. 6. При решении системы (8) для пика $\mathrm{Pt}(111)$ были использованы значения параметров аппаратной функции $b_{C}=0.03^{\circ}$ и $b_{G}=0.07^{\circ}$. Для пика $\operatorname{Pt}(222)$ использовались значения $b_{C}=0.07^{\circ}$ и $b_{G}=0.07^{\circ}$. Данные значения были получены в ре- зультате аппроксимации пиков эталона в окрестности углов $2 \theta=40^{\circ}$ (для пика $\operatorname{Pt}(111)$ ) и $2 \theta=85^{\circ}$ (для пика $\mathrm{Pt}(222))$ функциями Фогта методом наименьших квадратов.

Для исходных пленок $D$ примерно линейно увеличивается с ростом $h$, для пленок после отжига $D$ также увеличивается, но сублинейно (рис. 6,a). Отжиг всех исходных пленок приводит к увеличению $D$. Можно предположить, что обычные зерна, образовавшиеся, как и вторичные, в результате рекристаллизации, имеют низкую концентрацию дефектов, следовательно, их размер вдоль нормали к поверхности будет приближенно равен $D$. Таким образом, можно считать, что средняя высота обычных зерен будет, как и $D$, сублинейно расти с ростом $h$. В случае пленки $40 \mathrm{~nm}$ большинство зерен - обычные, поэтому $D$ приближенно равно средней высоте обычного зерна. Увеличение $D$ с ростом $h$ для исходных пленок можно объяснить уменьшением средней концентрации дефектов в них при увеличении толщины. Однако, так как исходные зерна могут содержать большое количество дефектов, высоту зерна в этом случае нельзя приравнивать к $D$. Поэтому наблюдаемое увеличение $D$ в результате отжига может происходить как на стадии рекристаллизации, так и на стадии роста зерен. Также во всех случаях отжиг приводит к значительному уменьшению $\varepsilon$, причем разность значений $\varepsilon$ до и после отжига слабо зависит от $h$. Значение данного параметра определяется концентрацией различных дефектов, поэтому можно предположить, что уменьшение микродеформаций решетки произошло, главным образом, на стадии рекристаллизации, однако дальнейшее их уменьшение на стадии роста зерен также нельзя исключать. 


\section{Заключение}

Таким образом, показано, что для Рt пленок с толщиной $h=20-100 \mathrm{~nm}$, подвергнутых отжигу в вакууме в режиме $500^{\circ} \mathrm{C} / 1 \mathrm{~h}$, скорость нормального роста зерен не зависит от $h$, а средний латеральный размер обычных зерен, так же как и исходных зерен, увеличивается с ростом $h$ линейно. Скорость аномального роста в соответствии с теорией увеличивается с уменьшением $h$ и начинает заметно превосходить скорость нормального роста при $h \sim 40 \mathrm{~nm}$. В результате распределение латеральных размеров зерен при $h=20-40 \mathrm{~nm}$ становится бимодальным, а средний латеральный размер вторичных зерен увеличивается с уменьшением $h$. Найдено, что распределения латеральных размеров исходных, обычных и вторичных зерен являются логнормальными. Размеры ОКР для исходной пленки увеличиваются с ростом $h$ линейно, для пленки после отжига - сублинейно. Для всех исходных пленок отжиг приводит к увеличению размеров ОКР и уменьшению микродеформаций.

Работа выполнена с использованием оборудования ЦКП „Диагностика микро- и наноструктур“ при финансовой поддержке Министерства образования и науки Российской Федерации.

\section{Список литературы}

[1] Izyumskaya N., Alivov Y.-I., Cho S.-J., Morcoc H., Lee H., Kang Y.-S. // Crit. Rev. Sol. State. 2007. Vol. 32. N 3-4. P. 111-202. doi: 10.1080/10408430701707347

[2] Воротилов К.А., Жигалина О.М., Васильев В.А., Сигов А.С. // ФТТ. 2009. Т. 51. Вып. 7. С. 1268-1271. (Vorotilov K.A., Zhigalina O.M., Vasil'ev V.A., Sigov A.S. // Phys. Sol. State. 2009. Vol. 51. N 7. P. 1337-1340. doi: 10.1134/S106378340907004X)

[3] Hong J., Song H.W., Lee H.C., Lee W.J., No K. // J. Appl. Phys. 2001. Vol. 90. N 4. P. 1962-1967. doi: 10.1063/1.1385358

[4] Matsumiya M., Shin W., Izu N., Murayama N. // Sens. Act. B. 2003. Vol. 93. N 1-3. P. 309-315. doi: 10.1016/S09254005(03)00223-5

[5] Ali M., Cimalla V., Lebedev V., Romanusa H., Tilak V., Merfeld D., Sandvik P., Ambacher O. // Sens. Act. B. 2006. Vol. 113. N 2. P. 797-804. doi: 10.1016/j.snb.2005.03.019

[6] Huang Z., Zhou W., Tang X. // Appl. Surf. Sci. 2010. Vol. 256. N 5. P. 2025-2030. doi: 10.1016/j.apsusc.2009.09.042

[7] Resnik D., Vrtacnik D., Mozek M., Pecar B., Amon S. // J. Micromech. Microeng. 2011. Vol. 21. N 2. P. 025025. doi: 10.1088/0960-1317/21/2/025025

[8] Thompson C.V. // J. Appl. Phys. 1985. Vol. 58. N 2. P. $763-$ 772. doi: $10.1063 / 1.336194$

[9] Wong C.C., Smith H.I., Thompson C.V. // Appl. Phys. Lett. 1986. Vol. 48. N 5. P. 335-337. doi: $10.1063 / 1.96543$

[10] Gruber W, Baehtz C., Horisberger M., Ratschinski I., Schmidt H. // Appl. Surf. Sci. 2016. Vol. 368. P. 341-347. doi: 10.1016/j.apsusc.2016.02.015

[11] Ruffino F., Grimaldi M.G., Bongiorno C., Giannazzo F., Roccaforte F., Raineri V., Spinella C. // J. Appl. Phys. 2009. Vol. 105. N 5. P. 054311. doi: $10.1063 / 1.3093681$
[12] Brongersma S.H., Richard E., Vervoort I., Bender H., Vandervorst $W$., Lagrange S., Beyer G., Maex K. // J. Appl. Phys. 1999. Vol. 86. N 7. P. 3642-3645. doi: 10.1063/1.371272

[13] Lingk C., Gross M.E., Brown W.L. // J. Appl. Phys. 2000. Vol. 87. N 5. P. 2232-2236. doi: $10.1063 / 1.372166$

[14] Платина, ее сплавы и композиционные материалы / Под ред. Е.В. Васильевой. М.: Металлургия, 1980. 296 с.

[15] Dannenberg R., Stach E.A., Groza J.R., Dresser B.J. // Thin Sol. Films. 2000. Vol. 370. P. 54-62. doi: 10.1016/S00406090(99)00947-5

[16] Briand D., Heimgartner S., Leboeuf M., Dadras M., de Rooij N.F. // Mater. Res. Soc. Symp. Proc. 2002. Vol. 729. U. 2.5. doi: 10.1557/PROC-729-U2.5

[17] Dai C.-L., Xiao F.-Y., Lee C.-Y., Cheng Y.-C., Chang P.-Z., Chang S.-H. // Mater. Sci. Eng. A. 2004. Vol. 384. N 1-2. P. 57-63. doi: 10.1016/j.msea.2004.05.067

[18] Yu H.Z., Thompson C.V. // Acta Materialia. 2014. Vol. 67. P. 189-198. doi: 10.1016/j.actamat.2013.12.031

[19] Jeffries J.H., Zuo J.-K., Craig M.M. // Phys. Rev. Lett. 1996. Vol. 76. N 26. P. 4931-4934. doi: 10.1103/PhysRevLett.76.4931

[20] Salvadori M.C., Melo L.L., Cattani M., Monteiro O.R., Brown I.G. // Surf. Rev. Lett. 2003. Vol. 10. N 01. P. 1-5. doi: 10.1142/S0218625X03004561

[21] Salvadori M.C., Brown I.G., Vaz A.R., Melo L.L.. Cattani M. // Phys. Rev. B. 2003. Vol. 67. N 15. P. 153404. doi: 10.1103/PhysRevB.67.153404

[22] Уманский Я.С., Скаков Ю.А., Иванов А.Н., Расторгуев Л.Н. Кристаллография, рентгенография и электронная микроскопия. М.: Металлургия, 1982. 632 с.

[23] Ruland W. // J. Appl. Cryst. 1968. Vol. 1. N 2. P. 90-101. doi: $10.1107 / \mathrm{S} 0021889868005066$

[24] Schoening F.R.L. // Acta Cryst. 1965. Vol. 18. N 5. P. 975-976. doi: $10.1107 / \mathrm{S} 0365110 \mathrm{X} 65002335$ 